\title{
Periventricular leucomalacia in premature infants in neonatal ward, Cipto Mangunkusumo Hospital: A preliminary study
}

\author{
Kemas Firman, MD; Idham Amir, MD; Nia Kurniati, MD; Sudigdo Sastroasmoro, MD, PhD
}

\begin{abstract}
Introduction Periventricular leucomalacia (PVL) is a major cause of neurodevelopment delay in premature infants, so early detection of the preterm infant at high risk for the subsequent development of this lesion is critical.

Objectives To determine the prevalence of PVL in premature infants hospitalized in neonatal ward, Cipto Mangunkusumo Hospital using cranial ultrasound scans and define its characteristics Methods Premature infants hospitalized in the neonatal ward from January to July 2003 were included in this study. Clinical features were retrieved from medical charts. Ultrasound scan was performed once, after the age of 7 days and interpretations were read separately by two consultants of the radiology division.

Results Fifty-one infants were included, 21 with $\leq 32$-week gestation, 30 with >32-week gestation; birth weight range were 1000 to 2600 gram. Nineteen infants had cystic lesion and/or dilatation of the ventricle from the cranial ultrasound scan. The prevalence of $P V L$ in gestational age (GA) of $\leq 32$ weeks was $6 / 21$ and that in GA of $>32$ weeks was $13 / 30$. Risk factors found in infants with PVL were maternal infection, respiratory distress, sepsis and circulatory failure.

Conclusion The prevalence of PVL in preterm infants in Cipto Mangunkusumo neonatal ward was higher than that was reported in developed countries. Maternal infection, respiratory distress, sepsis, and circulatory failure which were commonly found in these infants were factors to be considered as risks for PVL [Paediatr Indones 2004;44:117-122-126]
\end{abstract}

Keyword: premature infant, periventricular leucomalacia, prevalence, cranial ultrasound scan

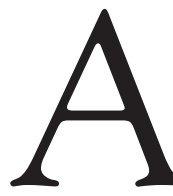

dvances in prenatal and neonatal care have significantly improved neonatal survival and decreased long-term morbidity, especially in infants born at $<32$-week gestation. These improvement are at least partly because of better understanding of neonatal diseases and improved care for extremely low birth weight infants ( $<1250$ grams). ${ }^{1,2}$ Extremely small newborn infants and those born too early are of special interest for medical, economical, social, and ethical reasons. In the United States premature birth accounted for $12 \%$ of all live birth. ${ }^{1}$ In our hospital, preterm births were up to $19 \%$ of all live births, and the smallest infant survivor had birth weight of 900 gram. ${ }^{3}$ Experts noticed that premature infants who survived have a higher possibility to have neurodevelopmental disability, especially cerebral palsy, visual or hearing disturbance, and learning difficulties. ${ }^{4-6}$ Hemorrhage, white matter damage (periventricular leucomalacia or PVL), and brain atrophy are some of the causes. ${ }^{7-11}$ Periventricular leucomalacia $(\mathrm{PVL})$ is a decrease in brain parenchyma. It appears on cranial ultrasonography as echoluscent lesions i.e., cavities or cysts within periventricular white matter. ${ }^{8,9}$ The pathophysiologic features of PVL are currently hypothesized to be multifactorial, including hypoxia/perfusion failure, genetic factors, and neurochemical-mediated injury to white matter (i.e., release of cytokines with infections or release of neurotransmitters e.g. glutamate with hypoxia). ${ }^{9-20}$

From the Department of Child Health, Cipto Mangunkusumo Hospital, Jakarta.

Request for reprints to: Kemas Firman, MD. Department of Child Health, Cipto Mangunkusumo Hospital, Jakarta. Tel. 62-21-3907740, Fax. 62$21-3907743$ 
Ultrasound has been used regularly to detect lesions in preterm brain, which can be done on very critical infants during their hospitalization. ${ }^{21,22}$ Neonatal centers in developed countries regularly perform cranial ultrasound scan with time protocol based on birth weight. ${ }^{17,23,24}$ American Academy of Neurology has outlined a protocol to evaluate preterm brain. ${ }^{25}$ Our hospital had limited facilities for delivering high care to high-risk infants, including imaging facilities. Until this study was conducted, PVL had never been recorded from our preterm survivors, which motivated us to investigate and find out the incidence of PVL in premature births.

\section{Methods}

This study was a cross sectional study. Ethical approval to study the premature infants' brain using ultrasound scan was given by the Research Ethics Committee, Medical School, University of Indonesia and parental consent was obtained for each subject. The patients were identified from the medical records of the neonatal ward, Cipto Mangunkusumo Hospital for deliveries between January and July 2003 at gestational age of less than 37 weeks with first-week hospitalization in our neonatal ward. Gestational age (GA) was determined according to the New Ballard Score ${ }^{26}$ and approved by the supervisor of the ward. Birth record was obtained from the medical chart of the infant. Infants who survived beyond the age of 7 days underwent cranial ultrasound studies, at least once (without a certain time protocol to follow).

\section{Ultrasound studies}

The ultrasound data collections were performed by 2 pediatric-radiologists who had kappa of 0.57 between them (moderately good). All sonographic studies were performed with a high-frequency transducer (Digital Gaia MT Sonoace 8800; 7.5 $\mathrm{MHz}$ ) and preserved for further assessment by hard copy and computer data (JPEG file). The data at least contain 3 frames of coronal and/or sagittal views; all used the anterior fontanel as the sonographic window. There was neither any timeset protocol to do the ultrasound data collection nor a detailed scanning procedure. All lesions were described as cysts or ventriculomegaly.

\section{Data retrieval}

The medical charts of the infants were reviewed for the presence or absence of the following characteristics including prolonged rupture of the membranes (PROM, defined as rupture for $\geq 18$ hours), pregnancy induced hypertension (defined as a systolic blood pressure of $>140 \mathrm{mmHg}$ and a diastolic blood pressure of $>90 \mathrm{mmHg}$, plus proteinuria with or without edema), multiple pregnancy, maternal infection (defined as maternal fever of $>38^{\circ} \mathrm{C}$, leukocytes of $>15,000 / \mathrm{ml}$, or signs of chorioamnionitis-like foul smelling discharge, uterine tenderness, and fever-), 1- and 5-minute Apgar scores, the need for delivery room resuscitation, birth weight, respiratory distress (defined as grunting, periodic apnea, retraction, hypoxia), the need for volume expanders with or without inotropic medications to support circulatory function in the neonatal period, evidence of sepsis, necrotizing enterocolitis (NEC), and patent ductus arteriosus (PDA) requiring either medical or surgical ligation.

A cystic lesion was defined either as echoluscent lesions observed in both coronal and sagittal views of periventricular area or ventricular dilatation with irregular lining. Echodense lesion was not included in this study because ultrasound examination was performed after 7 days of age.

\section{Consensus reading}

Each set of scan was read independently twice by two pediatric-radiologist consultant. Their interpretation was recorded separately. For final agreement individualized for each patient, the images were read together and final decision was made for each infant.

\section{Results}

\section{Subjects' characteristics}

Ninety-five infants were admitted to the neonatal ward with the diagnosis of prematurity. Gestational age was 
reaffirmed by the supervisor of the ward. Nine of these infants were term but small-for-date. Fifteen infants died before 7 days of age and 17 were missed to enter the study because they were discharged before 7 days of age or transferred to other hospitals. Until the end of the study, 1 infant did not undergo ultrasound scan and 2 sets of infant data were lost. Finally, we had 51 infant data to consider.

Thirty infants were of $>32$-week gestation $(59 \%)$ and 21 of $\leq 32$-week gestation. Birth weight ranged from 1000 to 2600 grams with 53\% (27/51 infants) had birth weight of $>1500$ grams. There were no data about maternal chorioamnionitis, although maternal infections were recorded in 27 infants (53\%). PROM was only found in 10/51 mothers. Asphyxia on 5-minute Apgar score affected only $11 / 51$ infants (22\%).

Recorded postnatal complications were respiratory distress, circulatory failure, cyanosis, sepsis, and hyperbilirubinemia. From all studied infants, respiratory distress affected $90 \%$ of the infants, while circulatory failure affected 69\% (35/51). Cyanosis was noted on 29\% of them (15/51). Sepsis with positive blood culture was documented in 32/51 (63\%) infants and hyperbilirubinemia affected almost $57 \%(29 / 51)$ of them.

\section{Cranial ultrasound findings}

Images were obtained in 51 infants, who, at the time of examination, had a median chronological age of 21 days (the earliest was 8 days, and the latest was 38 days). PVL lesions were observed in 19 (37\%) infants. The prevalence of PVL in infants with $\leq 32$ week-gestation was $6 / 21$ while in those with $>32$ week-gestation was $13 / 30$. It was noted that PVL was more frequent found in infants with GA of 35 weeks

Cystic lesions were noted in 15 infants, ventriculomegaly in 7 infants. Four infants had both ventriculomegaly and cystic lesions with irregular lining. Cystic lesions were recorded mostly in infants having ultrasound scan beyond the second week of life. Ventriculomegaly was seen as early as 8 days age. The size of the dilatation of the ventricle was not measured in this study. (Kappa's agreement between 2 reader after the study was 0.63 , which was categorized as good)

Other findings were intraparenchymal echodense that suggested the possibility of hemorrhage which was in accordance with the clinical appearance of the infants. This finding was found in 3 subjects. Cranial ultrasound scan performed in 2 weeks apart showed decreased area of hyperdensity.

\section{Clinical characteristics of infants with PVL}

Among 19 infants with PVL lesion, PROM was only found in 5 of them although maternal infection was found in 9 infants (Table 1). Respiratory distress, circulatory failure, and sepsis were found almost in all of them.
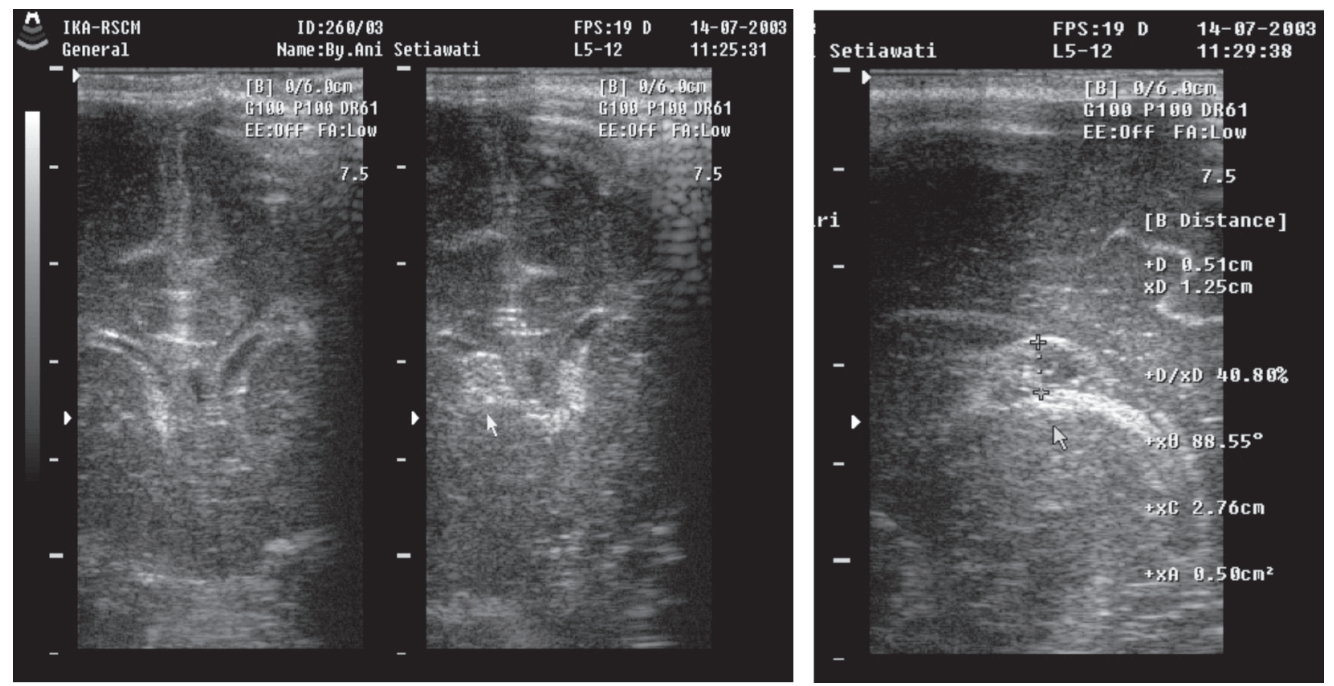

Figure 1. Cystic lesion in periventricular area 
Table 1. Characteristics of 19 infants With PVL

\begin{tabular}{|c|c|c|}
\hline Clinical features & $\begin{array}{l}\text { GA } \leq 32 \text { wk } \\
(n=6)\end{array}$ & $\begin{array}{l}\text { GA >32 wk } \\
(n=13)\end{array}$ \\
\hline Birth weight (mean) (grams) & 1437 & 1808 \\
\hline \multirow{2}{*}{$\begin{aligned} 5 \text {-minute Apgar score } & \leq 6 \\
& >6\end{aligned}$} & 2 & 4 \\
\hline & 4 & 9 \\
\hline Preeclampsia & 3 & 4 \\
\hline PROM & 1 & 4 \\
\hline Maternal infection & 4 & 5 \\
\hline Respiratory distress & 6 & 11 \\
\hline Circulatory failure & 4 & 10 \\
\hline Sepsis & 4 & 8 \\
\hline Hyperbilirubinemia & 3 & 6 \\
\hline Time of US (mean) (day) & 21 & 21 \\
\hline Image Cysts lesion & 3 & 12 \\
\hline Ventriculomegaly & 4 & 3 \\
\hline
\end{tabular}

US=ultrasound

In 4 infants with both cyst lesion and ventricular dilatation, one had a quite extensive lesion and his early clinical appearance was seen as spasticity of the lower extremities and right side arm. Figure 1 shows cystic lesions of this infant.

Two out of 19 infants died during hospitalization. Two infants showed spasticity of only lower extremities and 2 infants had feeding problem. Infants with PVL were sent to the outpatient clinic for longterm follow-up after hospital discharge.

\section{Discussion}

Only 54\% of the premature infants who were hospitalized in our hospital underwent cranial ultrasound scan. More than half of premature infants with GA of $<32$ weeks died in our hospital. The most common cause of death was infection, similar to that mentioned by Barton. ${ }^{5}$ Unfortunately chorioamnionitis was not recorded in the maternal medical chart, so that comparison could not be done with literature data. Perlman ${ }^{16}$ stated that chorioamnionitis and/or PROM history were important risk factors for preterm birth and subsequent PVL.

Maternal infection in this study was recorded higher compared to that of other study (9/19); it was probably the main cause of premature birth. The same condition was found in PROM (20\% of all infants compare to Perlman's ${ }^{16}$ data of $6 \%$ ). Based on these two conditions, the risk of infection in premature infants was high on our study.

Respiratory distress was found in 17 out of 19 infants and was managed without using mechanical ventilation because of lack of such facilities in our ward. This was higher than that reported by Fujimoto $^{15}(30.8 \%)$ or Perlman ${ }^{16}$ (22\%). Monitoring of distress or cyanosis in these infants was done by clinical observation indicating that mild ischemia was likely to be missed. According to Perlman observation and others working on the same problem, even such mild ischemia can be harmful to preterm brain. ${ }^{10,11,16,18}$

In this study, we found that the PVL prevalence for infant less than 32 week-gestation was

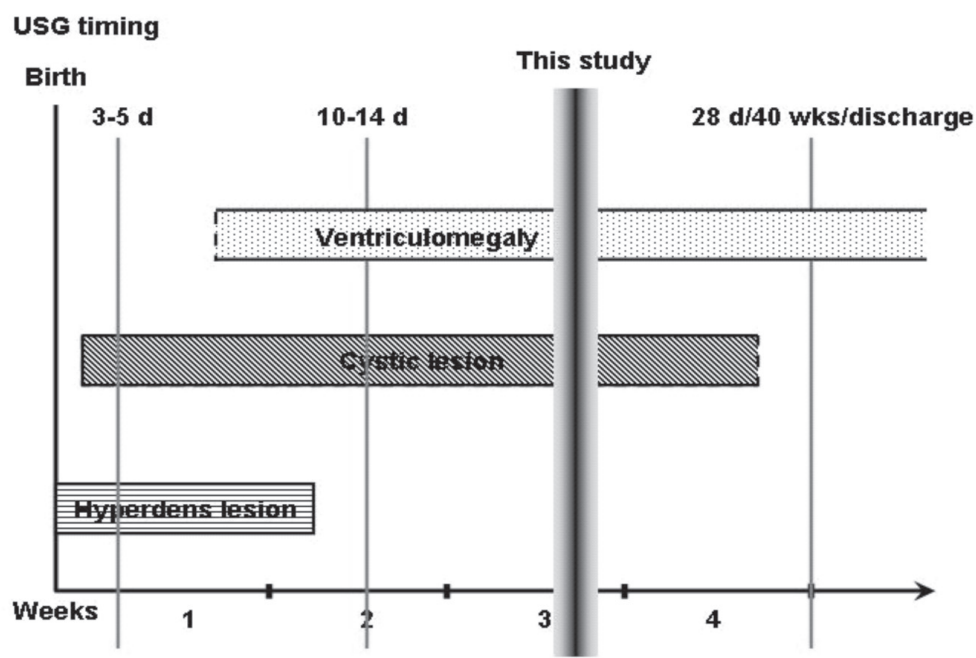

Figure 2. Timing of cranial ultrasound scans 
6/21, higher than reported by Murphy et al 23 (6.8\%) who worked on the same gestational age. It might be because the mortality was still high and infants who had chance to undergo cranial ultrasound scan were limited. The same condition was found in older infant. The occurence of PVL in preterm infants of $>32$-week gestation was $13 / 30$. It was higher than that was reported by Graziani et al ${ }^{14}(8.9 \%)$ or Fujimoto et al ${ }^{15}$ $(7.8 \%)$.

We did not expect to find hyperdense lesion such as mentioned by Volpe ${ }^{9}$ because ultrasound scan was performed after the age of first week. Protocol to screen premature infant had not been developed in our hospital and infant must be transported to the radiology unit to have an ultrasound scan. Screening protocol for preterm brain is well developed in some countries and long-term management can be delivered to ensure adequate growth and development. Figure 2 shows the comparisons of time protocol for performing cranial ultrasound scan in developed countries to that was used in this study.

We concluded that premature infants of less than 32-week gestation survived less than older preterm infants in our hospital. PVL as a consequence of brain insult was found higher than that was reported in literatures, both in infants of less than 32-week gestation and the older ones.

\section{References}

1. Sutton PD. Births, marriage, divorces and deaths: provisional data for January 2003. National Vital Statistics Reports. Volume 52 no.1. Maryland: Natural Center for Health Statistics; 2003.

2. Escobar GJ, Littenberg B, Petitti DB. Outcome among surviving very low birth weight infants: a meta-analysis. Arch Dis Child 1991;66:204-11

3. Subbagian Neonatologi Ilmu Kesehatan Anak FKUI/ RSCM. Morbiditas dan mortalitas neonatus di rumah sakit rujukan RSCM tahun 2001. Annual report. Unpublished.

4. Bucher HU, Ochsner Y, Fauchçre JC, Swiss Neonatal Network. Two years outcome of very preterm and very low birth weight infants in Switzerland. Swiss Med Wkly 2003;133:93-9
5. Barton L, Hodgman JE, Pavlova Z. Causes of death in the extremely low birth weight infant. Pediatrics 1999;103:406-51

6. Iriondo M, Alcover E, Lizarraga I, Campistrol J, Poo P, Ibanez M, et al. Sequelae prediction for cranial ultrasound in newborns < $1500 \mathrm{~g}$. RELAN 1999;1:123-8

7. Banker BQ, Larroche JC. Periventricular leukomalacia of infancy. Arch Neurol 1962;7:386-40

8. Bulas DI, Vezina GL. Preterm anoxic injury, radiologic evaluation. Radiol Clin N Am 1999;37:1146-62

9. Volpe JJ. Neurology of the newborn. 4th ed. Philadelphia: WB Saunders; 2001. p. 297-394

10. Subramaniam KNS. Pathophysiology of germinal matrix-related hemorrhage and ischemia. In: Grant EG, editor. Neurosonography of the preterm neonate. New York: Springer-Verlag; 1986. p. 25-32

11. Hill A, Volpe JJ. Hypoxic-ischemic cerebral injury in the newborn. In: Swaiman KF, Ashwal S, editors. Pediatric neurology, principles and practice. 3rd edition. Missoury: Mosby; 1999. p. 191-204

12. Volpe JJ. Intraventricular hemorrhage and brain injury in the premature infant neuropathology and pathogenesis. Clin Perinatol 1989;16:361-86

13. Armstrong D, Norman MG. Periventricular leucomalacia in neonates: complications and sequelae. Arch Dis Child 1974:49:367-75

14. Graziani LJ, Spitzer AR, Mitchell DG, Merton DA, Stanley C, Robinson N, et al. Mechanical ventilation in preterm infants: neurosonographic and developmental studies. Pediatrics 1992;90:515-22

15. Fujimoto S, Togari H, Yamaguchi N, Mizotani F, Suzuki S, Sobajima H. Hypocarbia and cystic periventricular leukomalacia in premature infants. Arch Dis Chil 1994;71:F107-10

16. Perlman JM, Risser R, Broyles RS. Bilateral cystic periventricular leukomalacia in the premature infant: associated risk factors. Pediatrics 1996;97:822-7

17. Perlman JM, Rollins N. Surveillance protocol for the detection of intracranial abnormalities in premature neonates. Arch Pediatr Adolesc Med 2000;134:822-6

18. Wiswell TE, Graziani LJ, Kornhauser MS, Stanley C, Merton DA, McKee L. Effects of hypocarbia on the development of cystic periventricular leukomalacia in premature infants treated with high-frequency jet ventilation. Pediatrics 1996;98:918-24

19. De Reuck J, Chattha AS, Richardson Jr.EP. Pathogenesis and evolution of periventricular leukomalacia in infancy. Arch Neurol 1972;27:229-40 


\section{Paediatrica Indonesiana}

20. Dammann O, Leviton A. Maternal intrauterine infection cytokines and brain damage in the preterm newborn [review]. Pediatr Res 1997;42:1-8

21. Paneth N, Rudelli R, Monte W, Rodriguez, Pinto J, Kairam $\mathrm{R}$, et al. White mater necrosis in very low birthweight infants: neuropathologic and ultrasonographic findings in infants surviving six days or longer. J Pediatr 1990;116:975-84

22. Slovis TL, Shankaran S. Ultrasound in the evaluation of hypoxic-ischaemic injury and intracranial hemorrhage in neonates: the state of the art. Pediatr Radiol 1984;14:67-75

23. Murphy DJ, Squier MV, Hope PL, Sellers S, Johnson A. Clinical association and time of onset of cerebral white matter damage in very preterm babies. Arch Dis Child 1996;75:F27

24. Boal DKB, Watterberg KL, Miles S, Gifford KL. Optimal cost-effective timing of cranial ultrasound screening in low birth-weight infants. Pediatr Radiol 1995;25:425-8

25. Ment LR, Bada HS, Barnes P, Grant PE, Hirtz D, Papile LA, et al. Practice parameter: neuroimaging of the neonate. Report of the quality standards subcommittee of the American Academy of Neurology and the practice committee of the Child Neurology Society. Neurology 2002;58:1726-38

26. Ballard JL, Khoury JC, Wedig K, Wang L, Eilers-Walsman BL, Lipp R. New Ballard score, expanded to include extremely premature infants. J Pediatr 1991;119:417-23 